\title{
ACCENT TONIQUE : SUBSTANCE(S) ET REPRÉSENTATION(S) Emilie Caratini
}

\author{
Armand Colin | « Langages »
}

2015/2 N 198 | pages 109 à 132

ISSN 0458-726X

ISBN 9782200929756

Article disponible en ligne à l'adresse :

https://www.cairn.info/revue-langages-2015-2-page-109.htm

\section{Pour citer cet article :}

Emilie Caratini, «Accent tonique : substance(s) et représentation(s) 》, Langages 2015/2 (N¹98), p. 109-132.

DOI 10.3917/lang.198.0109

Distribution électronique Cairn.info pour Armand Colin.

(C) Armand Colin. Tous droits réservés pour tous pays.

La reproduction ou représentation de cet article, notamment par photocopie, n'est autorisée que dans les limites des conditions générales d'utilisation du site ou, le cas échéant, des conditions générales de la licence souscrite par votre établissement. Toute autre reproduction ou représentation, en tout ou partie, sous quelque forme et de quelque manière que ce soit, est interdite sauf accord préalable et écrit de l'éditeur, en dehors des cas prévus par la législation en vigueur en France. Il est précisé que son stockage dans une base de données est également interdit. 


\section{Accent tonique : substance(s) et représentation(s)}

\section{INTRODUCTION}

Cet article s'intéresse à la/aux identité(s) ainsi qu'à la/aux représentation(s) qu'il est possible de conférer à un objet linguistique connu de tous : l'accent tonique (AT), i.e. la relative saillance de certaines syllabes dans la chaîne parlée. Par le biais de l'étude de plusieurs mécanismes, attestés dans la phonologie (synchronique ou diachronique) de l'allemand, et dont l'avènement est étroitement lié à la position de l'AT, il examine en allemand les effets que peut avoir l'AT sur les objets phonologiques qui l'entourent. Il est démontré que l'accent peut revêtir deux formes différentes pouvant cohabiter dans une même langue : il peut se manifester sous forme d'espace syllabique ou autoriser les voyelles phonologiquement longues à être réalisées comme telles sur le plan phonétique.

Après avoir rappelé la pertinence de l'accent dans l'analyse linguistique, la section 2 assied le besoin de discuter de l'identité phonologique de l'AT sur le fait que celui-ci porte une part de responsabilité dans l'accomplissement de processus de types parfois très hétérogènes. La section 3 propose la description de deux processus diachroniques (allongement vocalique [\$ 3.1.1] et consonantique [§ 3.1.2]) et de trois mécanismes synchroniques (occurrence du coup de glotte [§ 3.2.1], aspiration des occlusives non-voisées [§ 3.2.2] et exécution phonétique des voyelles longues [§ 3.2.3]) liés à l'accent et à sa position en allemand. Après avoir tracé les grandes lignes de la phonologie CVCV (cf. Lowenstamm 1996 ; Scheer 2004), la section 4 argumente en faveur de deux représentations de l'accent :

- une représentation en termes d'espace syllabique, capable de rendre compte :

- en diachronie, de l'allongement des voyelles toniques ou des consonnes immédiatement post-toniques (§ 4.2), si celui-ci est inséré à la droite de la voyelle tonique ; 
- en synchronie de l'allemand moderne, de l'occurrence du coup de glotte et de l'aspiration des occlusives non-voisées (§ 4.3.1), si celui-ci est inséré à la gauche de la voyelle frappée par l'accent ;

- et une manifestation diacritique (cf. Chomsky \& Halle 1968), qui promeut la réalisation phonétique de la longueur phonologique (§ 4.3.2).

La section 5 apporte quelques ultimes remarques sur la question de l'accent et de ses représentations.

\section{POURQUOI PARLER DE L'ACCENT TONIQUE ?}

\subsection{Une part de responsabilité dans divers phénomènes}

L'accent tonique joue un rôle important dans de nombreuses langues du monde appartenant à des groupes typologiquement et/ou génétiquement distincts. Concept avant tout phonético-phonologique, il a une part de responsabilité dans divers phénomènes linguistiques, dont certains, la majorité sans doute, relèvent purement du domaine phonético-phonologique, alors que d'autres, peut-être moins nombreux, peuvent également être liés à d'autres niveaux de l'analyse linguistique, par exemple à la (morpho)syntaxe. L'AT joue ainsi un rôle dans les phénomènes mentionnés dans la liste non exhaustive fournie dans le Tableau 1 (page suivante) ${ }^{1}$.

L'AT peut affecter des consonnes comme des voyelles ou même constituer un facteur de sélection sur le plan morphosyntaxique.

\footnotetext{
1. Si le français est pris en exemple dans le Tableau 1, ce n'est pas parce qu'il s'agit d'une langue à accent lexical, comme le sont par exemple le polonais (l'accent frappe de manière systématique l'avant-dernière syllabe du mot) et le tchèque (l'accent tombe invariablement sur la première syllabe du mot), mais parce que sa physionomie (comme celle des autres langues romanes) est en grande partie une conséquence directe de (la position de) l'accent tonique en latin. Il est communément admis qu'en français moderne l'accent tonique, dont l'intensité est par ailleurs moins importante que celle de l'accent tonique attesté dans d'autres langues (polonais, tchèque, anglais ou italien, etc.), ne semble pas lexical (i.e. ne semble pas être une propriété constitutive du signifiant du mot) et frappe la dernière syllabe d'une unité morphosyntaxique de taille variable pouvant correspondre au mot (notamment lorsque ce dernier est prononcé en isolation) ou à des unités de taille plus importante comme le syntagme ou même la phrase (cf. de Carvalho, Nguyen \& Wauquier, 2010 : 95-97). Certains chercheurs, dont Mertens (2009), distinguent un autre type d'accent en français moderne, un accent dit initial, qui peut se manifester de différentes manières.
} 
Tableau 1 : Processus liés à l'accent tonique a

\begin{tabular}{|c|c|}
\hline Phénomène et langue(s) concernée(s) & Description sommaire du phénomène \\
\hline $\begin{array}{l}\text { Réduction vocalique } \\
\text { (S[ynchronie]/D[iachronie]) } \\
\text { - anglais (cf. Chomsky \& Halle, } 1968: 38-39 \text { ) } \\
\text { - coratin (dialecte italien, cf. Bucci 2009; } \\
\text { D'Introno \& Weston 1997) } \\
\text { - latin > français (cf. Bourciez \& Bourciez, } \\
\text { 1967: } 31 \text { sqq.) } \\
\text { - russe (cf. Kenstowicz \& Kisseberth 1979) }\end{array}$ & $\begin{array}{l}\text { Hors accent, l'inventaire des voyelles dispo- } \\
\text { nibles est moins important (S) / les voyelles } \\
\text { s'amuïssent/chutent en position atone (D). }\end{array}$ \\
\hline $\begin{array}{l}\text { Allongement vocalique (S/D) } \\
\text { - anglais (cf. Minkova 1982) } \\
\text { - italien (cf. Vogel 1982) } \\
\text { - allemand (cf. Paul et al. 2007) } \\
\end{array}$ & $\begin{array}{l}\text { Les voyelles sont (S) / deviennent (D) plus } \\
\text { longues lorsqu'elles sont accentuées. }\end{array}$ \\
\hline $\begin{array}{l}\text { Loi de Verner (D) } \\
\text { - germanique (cf. Collinge } 1996 \text {; } \\
\text { Verner 1877) }\end{array}$ & $\begin{array}{l}/ f, \theta, h, h^{w} /\left(<\mathrm{PIE} / \mathrm{p}, \mathrm{t}, \mathrm{k}, \mathrm{k}^{\mathrm{w}} /\right) \text { et } / \mathrm{s} /(<\mathrm{PIE} / \mathrm{s} /) \\
\text { deviennent voisées sauf si elles sont en position } \\
\text { initiale de mot ou immédiatement précédées } \\
\text { d'une syllabe accentuée. }\end{array}$ \\
\hline $\begin{array}{l}\text { Diphtongaison (D) } \\
\text { - langues romanes (cf. Bourciez 1910) }\end{array}$ & $\begin{array}{l}\text { Certaines monophtongues, sous l'accent, } \\
\text { deviennent des diphtongues. }\end{array}$ \\
\hline $\begin{array}{l}\text { Flappisation (S) } \\
\text { - anglais américain (cf. Gussenhoven } 1986 \text {; } \\
\text { Harris } 1994 \text {; Kahn 1976) }\end{array}$ & $\begin{array}{l}/ t, d / \text { sont réalisés }[r] \text {; la syllabe suivant } / t, d / d o i t \\
\text { être atone. }\end{array}$ \\
\hline $\begin{array}{l}\text { Aspiration des occlusives non-voisées (S) } \\
\text { - anglais (cf. Kahn 1976) } \\
\text { - allemand (cf. Hall 1992, } 2000 \text {; } \\
\text { Wiese 1996) }\end{array}$ & $\begin{array}{l}\text { Les consonnes } / \mathrm{p} / \text {, } / \mathrm{t} / \text { et } / \mathrm{k} / \text { sont réalisées }\left[\mathrm{p}^{\mathrm{h}}\right] \text {, } \\
{\left[\mathrm{t}^{\mathrm{h}}\right] \text { et }\left[\mathrm{k}^{\mathrm{h}}\right] \text { notamment lorsqu'elles sont à l'ini- }} \\
\text { tiale d'une syllabe tonique. }\end{array}$ \\
\hline $\begin{array}{l}\text { Insertion du coup de glotte (S) } \\
\text { - allemand (cf. Alber } 2001 \text {; Hall 1992, } 2000 \text {; } \\
\text { Wiese 1996) } \\
\text { - français (cf. Fónagy } 1979 ; \text { Mertens 2009) }\end{array}$ & $\begin{array}{l}\text { Un coup de glotte est optionnellement inséré } \\
\text { à l'initiale de toute syllabe accentuée (d'une } \\
\text { syllabe frappée par l'accent initial en fran- } \\
\text { çais, cf. Mertens 2009) commençant par une } \\
\text { voyelle. }\end{array}$ \\
\hline $\begin{array}{l}\text { Allongement consonantique (S/D) } \\
\text { - italien (raddoppiamento fonosintattico, } \\
\text { cf. Vogel 1982) } \\
\text { - allemand [dialectes] } \\
\text { (cf. Schirmunski 1962; Seiler 2009) }\end{array}$ & $\begin{array}{l}\text { Certaines consonnes sont géminées lorsqu'elles } \\
\text { sont à proximité de l'accent. }\end{array}$ \\
\hline $\begin{array}{l}\text { Distribution des pronoms personnels (S) } \\
\text { - français (ex. Je le vois vs Lui, je le vois } \\
\text { vs *Le, je le vois) } \\
\text { - allemand (ex. Ich sehe inn 'je le vois' } \\
\text { vs Den sehe ich, mais* *lhn sehe ich) }\end{array}$ & $\begin{array}{l}\text { Seuls les pronoms personnels toniques peuvent } \\
\text { se trouver en position de focus ou de topic (en } \\
\text { gras) ; les pronoms atones doivent être adossés } \\
\text { à un verbe. }\end{array}$ \\
\hline
\end{tabular}

a. Dans le Tableau 1, S indique que le mécanisme concerné est d'ordre synchronique, D que le processus concerné est d'ordre diachronique, PIE signifie " proto-indo-européen ».

\subsection{Qu'est-ce que l'accent ?}

L'accent tonique, dont l'unité porteuse est la syllabe, rend la syllabe qu'il affecte plus proéminente que les autres syllabes d'un énoncé. Il peut être défini sur le plan phonétique ou phonologique. Si l'on considère la question sous un angle purement phonétique, une syllabe accentuée sera plus proéminente (perception) 
et fera l'objet d'un effort physique plus important que les autres syllabes (production, cf. Reetz \& Jongman, 2009 : 210-214). Elle se distinguera acoustiquement des autres syllabes le plus souvent par une fréquence fondamentale (F0) plus élevée et une durée plus importante, très souvent corrélées à d'autres particularités comme une plus grande intensité (cf. Ladd 1996 ; Ladefoged 2001 ; Reetz \& Jongman 2009 ; Roach 2001 ; Thomas, Bouquiaux \& Cloarec-Heiss 1976).

Si l'on adopte, en revanche, une perspective purement phonologique, l'AT, représenté par le diacritique ['] (ou []] dans le cas d'accents secondaires ${ }^{2}$, cf. Chomsky \& Halle 1968), est l'apanage des positions ou des syllabes prosodiquement fortes/saillantes (cf. Giegerich 1985), la relative force/saillance prosodique d'un élément pouvant être le reflet des relations morphosyntaxiques dans lesquelles l'unité portant l'accent est impliquée (cf. Nespor \& Vogel 2007 ; Scheer 2011). Il convient de distinguer deux types d'accents toniques (cf. Carvalho, Nguyen \& Wauquier, 2010 : 95-97, Thomas, Bouquiaux \& Cloarec-Heiss, 1976 : 103-104) :

- un accent, dit «libre", qui revêt une fonction distinctive dans certaines langues (cf. anglais record ['xeks:d] 'enregistrement' vs record [.II'ko:d] 'enregistrer') ;

- et un accent dit « fixe » qui aide à segmenter la chaîne parlée (fonction démarcative), et dont on peut prédire la position dans la chaîne parlée à l'aide d'une règle, comme c'est le cas en polonais, où l'accent frappe l'avant-dernière syllabe du mot.

Si la phonologie est capable, dans une certaine mesure, de prédire la place de l'AT dans une séquence donnée (à l'aide de règles ou d'autres mécanismes ; cf. Chomsky \& Halle, 1968 : 240) et propose des représentations pour les structures prosodiques responsables de la position de l'AT (cf. Nespor \& Vogel 2007) ou pour l'accent tonique lui-même (cf. Giegerich 1985 ; Liberman \& Prince 1977 ; Wiese, $1996: 272-280$ ), les manuels de phonologie ne s'attardent que rarement sur la substance de l'AT. Le plus souvent, ce dernier est simplement représenté à $l^{\prime}$ aide d'un diacritique ${ }^{3}$ pouvant revêtir différentes formes et dont on ne sait pas très bien pourquoi il serait la cause de processus aussi divers que ceux présentés dans le Tableau 1.

2. La littérature sur le sujet reconnaît trois niveaux d'accentuation différents et distingue les syllabes portant un accent principal/primaire (forte proéminence) des syllabes frappées d'un accent secondaire (proéminence moindre) et des syllabes non accentuées (pas de proéminence) (cf. Crystal 1994 ; Reetz \& Jongman 2009).

3. Le terme «diacritique» est ici employé au sens de Scheer (2005): la représentation de l'accent tonique dans le cadre de théories telles la phonologie prosodique (cf. Nespor \& Vogel 2007) ou métrique (cf. Giegerich 1985 ; Hayes 1990 ; Liberman \& Prince 1977, etc.) est diacritique dans la mesure où elle repose sur l'utilisation d'unités (pieds, mots prosodiques, etc.) organisées en une structure arborescente se voulant refléter les divisions morphosyntaxiques, mais qui sont en réalité construites sur la base des effets phonologiques qu'elles engendrent. Par comparaison, la structure syllabique est étroitement liée aux propriétés phonologiques des éléments qu'elle enferme (et non à leurs propriétés morphosyntaxiques) : la syllabe n'est donc pas diacritique contrairement aux unités de rang plus élevé dans la hiérarchie prosodique. 
Quelle est donc la substance de l'AT, quelle est donc son essence ? Afin d'apporter une réponse à cette question, la section suivante propose l'étude de cinq phénomènes diachroniques et synchroniques impliquant l'accent tonique, attestés en allemand (standard et/ou dialectal) : allongement vocalique, allongement consonantique, présence $\mathrm{d}^{\prime}$ une attaque glottale, aspiration et réalisation des voyelles phonologiquement longues.

\section{L'ACCENT EN ALLEMAND : FAITS}

En allemand, la position de l'AT, qui est de nature essentiellement démarcative ${ }^{4}$, peut être prédite : dans les racines d'origine germanique, il se trouve sur la première syllabe du radical (cf. Paul, 1968 : § 17) ; la situation est quelque peu différente dans les formes $d^{\prime}$ origine étrangère (l'accent est par exemple final dans la grande majorité des emprunts récents au français, ex. restaurant [вєsto'ьа̃] 'restaurant'), dans lesquelles la fonction démarcative de l'AT reste toutefois manifeste.

Quelle que soit l'origine des formes concernées, l'AT a par ailleurs une part de responsabilité dans divers mécanismes à l'œuvre dans la phonologie de l'allemand. Certains d'entre eux, qui peuvent être de nature diachronique ou synchronique, sont examinés dans les sections suivantes.

\subsection{Diachronie}

L'AT a joué un rôle capital dans l'histoire récente de l'allemand ${ }^{5}$ : dans certains contextes, il a contribué à l'allongement des voyelles brèves ainsi qu'à l'allongement de consonnes simples.

\subsubsection{Allongement vocalique}

Tout comme d'autres langues germaniques à la même période, l'allemand a subi un processus d'allongement vocalique entre le $\mathrm{XII}^{\mathrm{e}}$ et le $\mathrm{XIV}^{\mathrm{e}}$ siècles, processus qui a affecté exclusivement les voyelles brèves accentuées, comme le démontrent les exemples dans le Tableau 2 (cf. Paul 1884 ; Paul et al. 2007 ; Russ 1969).

\footnotetext{
4. À l'exception de quelques cas de figure dans lesquels il lui arrive de revêtir un caractère distinctif, cf. übersetzen ['y:bezetsn] 'faire passer sur l'autre rive' vs. übersetzen [ybe'zetsn] 'traduire'. Cet article s'intéresse uniquement à l'accent tonique primaire, la présence d'accents secondaires étant due à la computation morphosyntaxique et étroitement liée à la position des accents primaires ainsi qu' aux schémas accentuels induits par certains morphèmes dérivationnels. Par ailleurs, l'analyse présentée ici propose des représentations phonologiques de l'accent et s'intéresse à sa substance ; elle n'est donc aucunement incompatible avec les études sur la position de l'accent menées dans le cadre d'autres théories (phonologie prosodique, phonologie métrique, etc.), qui se préoccupent de la prédiction des schémas accentuels dans les séquences morphologiquement complexes et requièrent donc une/des théorie(s) de l'interface entre la phonologie et la (morpho)syntaxe.
}

5. Plus précisément lors de la transition entre le MHA (moyen haut allemand, 1050-1350) et le NHA (nouveau haut allemand, depuis 1650). 
Tableau 2 : Allongement vocalique : sous l'accent uniquement

\begin{tabular}{|c|c|c|c|}
\hline & MHA & NHA & Gloses \\
\hline \multirow{5}{*}{ 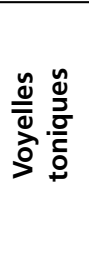 } & $\operatorname{sig}(e)$ & $S[i:] g$ & victoire \\
\hline & wec & $W[\mathrm{e}:] g$ & chemin \\
\hline & termin & Term[i:]n & rendez-vous \\
\hline & sun & S[o:]hn & fils \\
\hline & ber & $B[\mathrm{e}:] r$ & ours \\
\hline \multirow{5}{*}{$\begin{array}{l}\tilde{y} \\
\overline{\overline{0}} \\
\stackrel{y}{0} \\
\stackrel{0}{0} \\
\stackrel{0}{0}\end{array}$} & $-i g$ & $-[1] g$ & suffixe ADJ \\
\hline & wec & $w[\varepsilon] g$ & parti \\
\hline & bin & $b[1] n$ & (je) suis \\
\hline & un-a & {$[v] n-$} & préfixe privatif \\
\hline & er- & {$[\varepsilon] r-$} & préfixe (achèvement) \\
\hline
\end{tabular}

a. Un relecteur anonyme note, à juste titre, que le préfixe un- est souvent tonique en NHA. Cependant, ce n'est pas du caractère tonique vs atone des voyelles du NHA qu'il s'agit, mais bien de celui des voyelles du MHA : en effet, ce sont les propriétés prosodiques des voyelles du MHA qui ont conditionné l'évolution de ces dernières, et non les propriétés prosodiques des voyelles du NHA. La particule inséparable du MHA un- est présentée dans Müller \& Zarncke (2007 : 182) comme ne portant pas l'accent (principal) dans la majorité des cas, car celui-ci affecte la première syllabe de la racine à laquelle elle est rattachée.

Par ailleurs, l'allongement vocalique ne s'est produit que dans certains contextes (cf. Paul et al. 2007 ; Ritzert 1898).

Tableau 3 : Allongement vocalique : contextes ${ }^{\text {a }}$

\begin{tabular}{|c|c|c|c|c|}
\hline Contexte & MHA & NHA & Gloses & Allongement ? \\
\hline a. $-C \mathrm{CV}$ & vinden & $f[1]$ nden & trouver & \multirow{2}{*}{ Non } \\
\hline - C C\# & alt & [a]/t & vieux & \\
\hline \multirow{2}{*}{ b. $\_C(C) V$} & vater & $V[a:] t e r$ & père & \multirow{2}{*}{ Oui } \\
\hline & sigrist(e) & S[i:]grist & sacristain & \\
\hline c. _ C \# & wol & $w[0:] h l$ & bien & Oui \\
\hline d. _V & sehen & s[e:]hen & voir & Oui \\
\hline e. _\# & ne & $n[\mathrm{e}:]$ & non & Oui \\
\hline
\end{tabular}

a. C C indique ici la présence de consonnes dont la première au moins appartient à la même syllabe que la voyelle qui précède. $C(C)$ correspond à un cluster [groupe de consonnes] dans lequel les deux consonnes sont associées à la voyelle qui suit.

Les voyelles brèves accentuées du MHA deviennent longues si et seulement si elles précèdent une consonne simple intervocalique (b.) ou finale (c.), une autre voyelle (d.) ainsi que lorsqu'elles se trouvent en finale absolue de mot (e.). Aucun allongement ne se produit si la voyelle tonique est suivie de plus d'une consonne non-finale (a.). 
Il existe par ailleurs des cas dans lesquels, pour des raisons relativement obscures sur le plan strictement phonologique, une voyelle brève se trouvant dans un contexte adéquat en MHA ne devient pas longue en NHA ${ }^{6}$. De tels cas sont recensés chez V. Schirmunski (1962 : 270 sqq.) et H. Paul (1968 : § 247), qui notent que, lorsque la voyelle ne devient pas longue, c'est la consonne suivante qui le devient.

\subsubsection{Allongement consonantique}

Le processus d'allongement consonantique observé par V. Schirmunski et $\mathrm{H}$. Paul, qui est présenté comme complémentaire au processus d'allongement vocalique attesté par ailleurs, est spécifique à certains dialectes de l'allemand supérieur, plus précisément au souabe (sud-ouest de l'Allemagne), au bavarois du sud (extrême sud de l'Allemagne, sud de l'Autriche, nord de l'Italie) et au haut alémanique (extrême sud-ouest de l'Allemagne, Suisse).

Ainsi, alors que l'allemand standard exhibe des voyelles longues et des consonnes brèves dans des formes telles que N[a:]me 'nom' (< MHA name), $W[o:] h l$ 'bien' (<MHA wol(e)), n[e:]hmen 'prendre' (<MHA nemen) et $V$ [a:]ter 'père' (< MHA vater), certains dialectes de l'allemand supérieur possèdent des voyelles brèves et des consonnes longues dans les formes correspondantes (cf. Tableau 4).

Tableau 4 : Allongement consonantique : souabe et haut alémanique (Schirmunski 1962)

\begin{tabular}{|c|c|c|}
\hline MHA & NHA/dialectes & Gloses \\
\hline name & $n a^{n} m m e$ & nom \\
\hline wol(e) & woll & bien \\
\hline nemen & $n e^{n} m m e$ & prendre \\
\hline vater & fatter & père \\
\hline
\end{tabular}

Phonétiquement, la durée de ces consonnes, brèves en MHA et devenues longues lors de la transition vers le NHA, est par ailleurs identique à celle des géminées héritées du MHA dans ces dialectes (cf. Schirmunski, 1962 : 270-271).

La situation de l'allemand standard est a priori moins limpide que celle de l'allemand dialectal : sur le plan phonétique, il n'existe en effet pas de géminées

\footnotetext{
6. En allemand standard, il s'agit principalement de formes dans lesquelles la voyelle tonique, brève, précède une obstruante non-voisée se trouvant entre deux voyelles ou en finale absolue de mot (ex. MHA blat $>$ NHA $B l[a] t t$ 'feuille'). De nombreux exemples similaires impliquant des sonantes sont aussi attestés (ex. MHA doner $>$ NHA $D[$ o]nner 'tonnerre'). Très rares sont les cas de figure impliquant une obstruante voisée intervocalique (ex. MHA wider > NHA W[I]dder 'bélier') et aucun exemple ne met en cause une obstruante voisée finale. (cf. Caratini 2009 pour davantage de détails sur la question). Tout cela montre que l'identité de la consonne post-vocalique détermine en partie le devenir des voyelles toniques brèves.
} 
dans la langue standard ${ }^{7}$, où même la morphologie, qui peut faire se rencontrer deux phonèmes consonantiques identiques, ne permet pas la production de consonnes phonétiquement longues/géminées (ex. ent+täuschen 'décevoir'

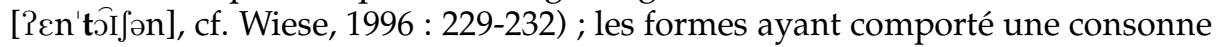
géminée en MHA n'affichent que des consonnes simples en NHA (ex. MHA mitte > NHA Mi[t]e 'milieu').

Tout cela indique qu'un processus de dégémination a affecté certains dialectes allemands, notamment ceux ayant servi de socle à la standardisation de $l^{\prime}$ allemand, entre le MHA et le NHA ${ }^{8}$. Ce processus, en revanche, semble ne pas avoir atteint les dialectes du souabe, du bavarois du sud et du haut alémanique mentionnés supra, qui possèdent toujours des consonnes géminées. On peut déduire de cela que l'ensemble des dialectes du haut allemand a très probablement connu le processus d'allongement consonantique complémentaire à l'allongement vocalique, processus dont les effets ne sont plus perceptibles aujourd'hui en allemand standard à cause de la dégémination intervenue par la suite (cf. Caratini, 2009 : 444 sqq.).

L'accent a donc joué un rôle important dans la diachronie de l'allemand standard et dialectal en ce qui concerne le processus d'allongement vocalique et consonantique.

\subsection{Synchronie}

En allemand moderne, l'accent a une part de responsabilité dans trois mécanismes synchroniquement actifs discutés infra : réalisation d'une attaque glottale à l'initiale de certaines syllabes commençant par une voyelle (§ 3.2.1), aspiration des occlusives sourdes (§ 3.2.2) et réalisation des voyelles longues (§ 3.2.3).

\subsubsection{Attaque glottale [?]}

Comme le montrent les données présentées infra, une attaque glottale est réalisée à l'initiale de syllabes commençant par une voyelle quand celles-ci sont accentuées (cf. Tableau 5), ou se trouvant en début de morphème (cf. Tableau 6) ${ }^{9}$.

7. Si l'orthographe allemande dispose bien de géminées graphiques, celles-ci ne reflètent aucunement la prononciation (ex. Blatt 'feuille' prononcé ['blat], cf. Hall, 1992 : 50).

8. Comme le souligne un relecteur anonyme, un processus de dégémination était déjà présent en vieux haut allemand (750-1050). Ce processus de dégémination a cependant affecté les géminées dans certains contextes seulement : lorsqu'elles étaient précédées d'une voyelle longue ou d'une diphtongue, lorsqu'elles se trouvaient en fin de mot ou en position préconsonantique (cf. Paul, 1968 : § 247 ; Braune \& Reiffenstein, 2004 : § 93). Le processus de dégémination, dont il est question ici, est beaucoup plus général et a affecté la totalité des géminées de l'allemand, y compris les géminées (anciennes, i.e. héritées du MHA, et nouvelles, i.e. apparues après le MHA, intervocaliques ou finales) précédées d'une voyelle brève.

9. Le coup de glotte est la seule consonne dont la présence est régulée phonologiquement en allemand : il ne s'agit donc pas d'un phonème. Les exemples sont tirés de Caratini (2009 : 75). 
Tableau 5 : Attaque glottale : syllabes accentuées

\begin{tabular}{|c|c|}
\hline Formes & Gloses \\
\hline $\begin{array}{l}\text { Theater }\left[\mathrm{t}^{\mathrm{h}} \mathrm{e}^{\prime} \mathrm{Pa}: \mathrm{te}\right] \\
\left.\text { mais : theatralisch [t }{ }^{\mathrm{h}} \text { ea't } \chi \mathrm{a}: \mathrm{II} \int\right]\end{array}$ & $\begin{array}{l}\text { théâtre } \\
\text { théâtral(e) }\end{array}$ \\
\hline $\begin{array}{l}\text { Oase [?o'?a:Zə] } \\
\text { mais : fähig ['fe:ıç] }\end{array}$ & $\begin{array}{l}\text { oasis } \\
\text { capable }\end{array}$ \\
\hline $\begin{array}{l}\text { Ukraine [?uk } \chi a^{\prime} \text { 'i:nə] } \\
\text { mais : Eozän [?eo'tse:n] }\end{array}$ & $\begin{array}{l}\text { Ukraine } \\
\text { Éocène }\end{array}$ \\
\hline
\end{tabular}

Tableau 6 : Attaque glottale : syllabes initiales de morphème (périphérie gauche seulement)

\begin{tabular}{|c|c|}
\hline Formes & Gloses \\
\hline $\begin{array}{l}\text { ent-täuschen [?en'tôljən] } \\
\text { mais : Bau-er ['bâve] }\end{array}$ & $\begin{array}{l}\text { décevoir } \\
\text { paysan }\end{array}$ \\
\hline $\begin{array}{l}\text { über-einander ['?ybe?âl'nande] } \\
\text { mais : geh-en ['ge:ən] }\end{array}$ & $\begin{array}{l}\text { I'un sur l'autre } \\
\text { aller }\end{array}$ \\
\hline $\begin{array}{l}\text { Oase [?o'Pa:Zə] } \\
\text { mais : zieh-en ['ți:ən] }\end{array}$ & $\begin{array}{l}\text { oasis } \\
\text { tirer }\end{array}$ \\
\hline
\end{tabular}

Bien évidemment, un coup de glotte est également réalisé dans les positions cumulant les deux contextes mentionnés supra (i.e. en début de syllabe initiale accentuée, cf. Tableau 7).

Tableau 7 : Attaque glottale en début de syllabe initiale accentuée

\begin{tabular}{|c|c|}
\hline Formes & Gloses \\
\hline $\begin{array}{l}\text { Amt ['Pamt] } \\
\text { mais : Pfeiler ['pfâlle] }\end{array}$ & $\begin{array}{l}\text { office } \\
\text { pilier }\end{array}$ \\
\hline 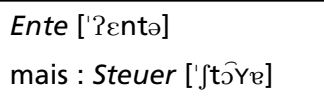 & $\begin{array}{l}\text { canard } \\
\text { taxe }\end{array}$ \\
\hline 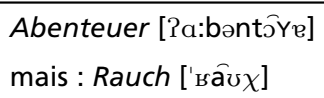 & $\begin{array}{l}\text { aventure } \\
\text { fumée }\end{array}$ \\
\hline
\end{tabular}

L'occurrence du coup de glotte est toutefois sujette à variation (sociolinguistique et géographique). La présence d'un coup de glotte est obligatoire en début de mot, mais reste facultative dans les hiatus.

\subsubsection{Aspiration des consonnes}

Un deuxième phénomène sensible à l'accent est l'aspiration des occlusives non-voisées $/ \mathrm{p} /, / \mathrm{t} /$ et $/ \mathrm{k} /$ de l'allemand. La littérature sur le sujet est assez riche, et, si les phonologues ayant travaillé sur la question débattent encore de la représentation mélodique à allouer à ces consonnes (en termes de traits 
distinctifs, par exemple ; cf. Bluhme 1970 ; Jessen 1998 ; Seiler 2004), ils semblent $\mathrm{s}^{\prime}$ accorder sur le fait que les phonèmes $/ \mathrm{p} /, / \mathrm{t} /$ et $/ \mathrm{k} /$ de l'allemand sont aspirés si et seulement $\mathrm{s}^{\prime}$ ils se trouvent dans un des deux contextes suivants (cf. Iverson \& Salmons 1995) :

- en début de mot (ex. Theater [ $\mathrm{t}^{\mathrm{h}} \mathrm{e}$ 'Pa:te] 'théâtre') ;

- et/ou en début de syllabe accentuée (ex. Kartoffel $\left[\mathrm{k}^{\mathrm{h}} \mathrm{\alpha}: \mathrm{t}^{\mathrm{h}} \mathrm{\partial fəl}\right.$ ] 'pomme-deterre').

$/ \mathrm{p} /, / \mathrm{t} /$ et $/ \mathrm{k} /$ sont bien évidemment aussi aspirés quand ils se trouvent en début de syllabe initiale accentuée (ex. Kind [' $\left.\mathrm{h}^{\mathrm{h}} \mathrm{Int}\right]$ 'enfant').

L'aspiration de $/ \mathrm{p} /, / \mathrm{t} /$ et $/ \mathrm{k} /$ semble donc se produire exactement dans les contextes identifiés comme pertinents lorsqu'il s'agit de prédire l'occurrence du coup de glotte (§3.2.1).

\subsubsection{Exécution phonétique des voyelles longues}

Le dernier phénomène sensible à l'accent tonique dont il sera question dans cet article affecte le système vocalique de l'allemand moderne. Si l'accent tonique de l'allemand a été présenté comme ayant une fonction démarcative - et comme étant associé de façon systématique à la première syllabe des racines dans les formes d'origine germanique - dans la section 3, cela ne signifie pas pour autant $\mathrm{qu}^{\prime}$ il est stable dans tous les paradigmes. Certain affixes sont ainsi à même de faire varier la position de l'accent tonique (cf. Theater [ $\mathrm{t}^{\mathrm{h}} \mathrm{e}$ '?a:te] 'théâtre' vs. theatralisch [ $\mathrm{t}^{\mathrm{h}}$ ea't $\left.\chi \mathrm{a}: 1 \mathrm{I}\right]$ ] 'théâtral'), et certaines formes peuvent tolérer deux schémas accentuels (variation géographique).

Par ailleurs, l'allemand moderne, comme l'on peut s'y attendre, possède des voyelles brèves et des voyelles longues, dont l'occurrence ne peut être prédite par le biais d'une règle, comme le montre l'existence des paires minimales fournies dans le Tableau 8. L'opposition longue $v$ s brève dans le système vocalique de l'allemand n'est du reste pas une spécificité des formes d'origine germanique et s'étend à l'ensemble du lexique de l'allemand.

Tableau 8 : NHA : quelques paires minimales

\begin{tabular}{|l|l|}
\hline Voyelle longue & Voyelle brève \\
\hline Miete ['mi:tə] 'loyer' & Mitte ['mıtə] 'milieu' \\
\hline Beet ['be:t] 'platebande' & Bett ['bet] 'lit' \\
\hline Höhle ['hø:lə] 'cave' & Hölle ['hœlə] 'enfer' \\
\hline Aal ['?a:l] 'anguille' & all ['Pal] 'tout' \\
\hline eben ['Pe:bən] 'plat' & ebben ['?ebən] 'baisser (marée)' \\
\hline
\end{tabular}

Bien que la longueur vocalique de l'allemand moderne soit phonémique, il est intéressant de constater que, lorsqu'une voyelle, longue sous l'accent, se retrouve atone pour des raisons morphologiques, elle n'est pas réalisée phonétiquement comme une voyelle longue (cf. Tableau 9). 
Tableau 9 : NHA : variations de longueur vocalique par rapport à l'accent

\begin{tabular}{|c|c|}
\hline Voyelle sous l'accent & Voyelle hors accent \\
\hline Möbel ['mø:bəl] 'meuble' & möb/+ieren [mø'bli::ьən] 'meubler' \\
\hline Theater [th e'?a:te] 'theâtre' & theatr+alisch [ $\mathrm{t}^{\mathrm{h}}$ ea't $\left.\chi \mathrm{a}: \mathrm{IIJ}\right]$ 'théâtral' \\
\hline Zug ['tsu:k] 'train' & $A n+z u g$ ['Pantsuk] 'costume' \\
\hline Motor ['mo:toe] 'moteur' & Motor+en [mo't ${ }^{\mathrm{h}}$ о:вәn] 'moteurs' \\
\hline Japan ['ja:pan] 'Japon' & Japan+er [ja'pha:ne] 'japonais' \\
\hline Oboe [?o'bo:ə] 'hautbois' & Obo+ist [?obo'?Ist] 'hautboïste' \\
\hline Anis [?a'ni:s] 'anis' & Anis ['?a:nis] 'anis' \\
\hline Gisela ['gi:zela] (prénom) & Gisela [gi'ze:la] (prénom) \\
\hline
\end{tabular}

Nous pouvons déduire de cela que la présence de l'accent est requise, dans les mots d'origine germanique comme dans ceux d'origine étrangère, pour qu'une voyelle phonologiquement longue soit réalisée comme telle sur le plan phonétique.

Il est important de souligner ici que, même si les voyelles potentiellement longues sont phonétiquement brèves lorsqu'elles ne se trouvent pas en syllabe accentuée (ex. Motoren [mo't ${ }^{\mathrm{h}} \mathrm{o}$ :เən] 'moteurs'), elles ne peuvent pour autant être confondues avec des voyelles phonologiquement brèves : l'opposition longue vs brève est en effet doublée d'une opposition de timbre en allemand ; la majorité des voyelles phonologiquement longues ([i:], [y:], [u:], [e:], [ø:] et [o:]) sont également tendues, alors que les voyelles brèves $([\mathrm{I}],[\mathrm{Y}],[\mho],[\varepsilon],[œ]$ et $[\supset])$ sont relâchées ${ }^{10}$.

\subsection{Conclusion intermédiaire}

Les paragraphes précédents ont contribué à démontrer que la présence de l'accent tonique est nécessaire à l'avènement de certains processus phonologiques, qui peuvent être de nature diachronique (§ 3.1) ou synchronique (§ 3.2), et peuvent affecter les consonnes comme les voyelles.

La section suivante, après avoir présenté le cadre théorique de la phonologie CVCV (cf. Lowenstamm 1996 ; Scheer 2004) et en considérant les effets que l'accent tonique peut avoir sur son environnement, sera consacrée à la/aux représentation(s) phonologique(s) qu'il est possible de faire de l'accent.

10. Il existe également une différence de timbre assez nette en ce qui concerne les voyelles basses de l'allemand, /a:/ (postérieure) et /a/ (antérieure), mais celle-ci ne relève pas à proprement parler de la tension vocalique impliquée dans l'articulation de ces deux voyelles. Deux voyelles appartenant au noyau dur du système phonologique de l'allemand ont été ignorées jusqu'ici : /ə/, qui n'est présent qu'en syllabe atone, et / $\varepsilon$ :/, dont l'existence-même est controversée, et qui est prononcée [e:] ou [ $\varepsilon$ ] par les locuteurs natifs (cf. Moulton, $1947: 213$; Wiese, $1996: 17)$. 


\section{QUELLE(S) REPRÉSENTATION(S) POUR L'ACCENT ?}

\subsection{Cadre théorique : la phonologie CVCV}

La phonologie CVCV, qui procède de la phonologie de gouvernement (cf. Kaye 1990 ; Kaye, Lowenstamm \& Vergnaud 1990), est une théorie phonologique relativement récente, née vers la fin des années 1990 (cf. Lowenstamm 1996). Nous nous attacherons ici à résumer les grandes lignes de son fonctionnement, qui est présenté en détail dans T. Scheer (2004).

La phonologie CVCV, contrairement à d'autres théories phonologiques (cf. Goldsmith, Riggle \& Yu, 2011 : 164-196), considère que la chaîne phonologique consiste en une stricte alternance de positions consonantiques (C) et vocaliques $(\mathrm{V})$, i.e. en une stricte consécution d'unités CV (l'unité CV étant le seul constituant syllabique pouvant être manipulé par la phonologie), et que les différences de structure syllabique observables en surface ne sont pas le fait de structures arborescentes mais la conséquence de relations latérales qui s'exercent entre les positions vocaliques et consonantiques. Toute séquence phonologique, qu'il s'agisse de séquences simples de type CV (cf. français $m a$ ) ou de séquences plus complexes telles que le français avatar, crabe ou opter, sera ainsi analysée au niveau sous-jacent comme une stricte consécution d'unités CV.

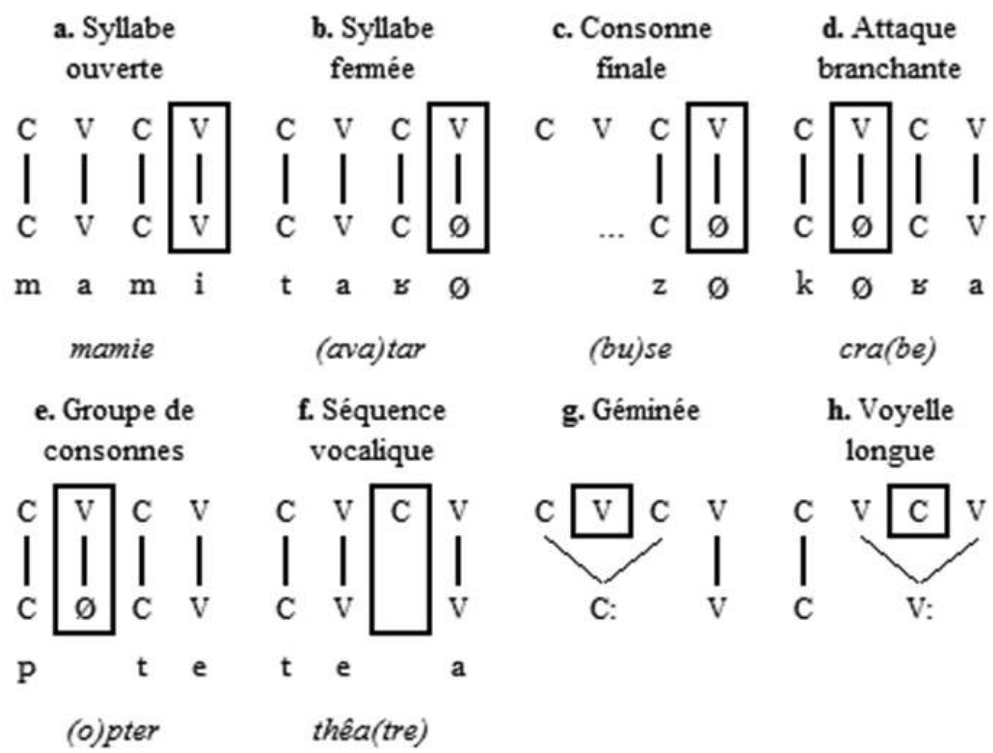

Figure 1 : Phonologie CVCV : quelques représentations 
Pour représenter des séquences plus complexes que celles de type $\mathrm{CV}$, la phonologie $\mathrm{CVCV}$ doit recourir à des positions, consonantiques ou vocaliques, vides $(\emptyset)$, dont l'occurrence est régulée par le biais de deux relations latérales antagonistes : le licenciement (Lic.), qui promeut l'expression mélodique de sa cible, et le gouvernement (Gouv.), qui inhibe l'expression segmentale de sa cible (cf. Ségéral \& Scheer, 2001 : 138). Par ailleurs, toute position (vocalique) non exprimée doit être gouvernée par une position vocalique à sa droite ou par le biais du gouvernement, paramétrique, des noyaux vides finaux. Certaines positions, pour l'essentiel vocaliques ${ }^{11}$, sont donc capables d'exercer gouvernement et/ou licenciement sur leur entourage. Ces deux forces sont monodirectionnelles (gauche $\leftarrow$ droite) et font entrer en relation des éléments nécessairement adjacents (à un niveau donné) ${ }^{12}$.

Enfin, la capacité de gouvernement/licenciement des différents types de positions, et notamment des différents types de positions vocaliques, est pour partie paramétrique, i.e. spécifique à chaque langue :

- les positions vocaliques exprimées/noyaux pleins stables ${ }^{13}$ (NP) sont universellement aptes à gouverner et/ou licencier ;

- les positions vocaliques internes non-exprimées/noyaux vides internes (NVI) sont universellement de piètres licencieurs et de piètres gouverneurs ;

- chaque langue détermine, en revanche, la capacité de licencier et/ou de gouverner des positions vocaliques finales non-exprimées/noyaux vides finaux $(\mathrm{NVF})$.

\subsection{Diachronie}

Sur le plan diachronique, dans certaines conditions relatives à la structure syllabique, il se produit deux événements en allemand : allongement vocalique et allongement consonantique. Sur le plan représentationnel, cela revient à faire d'une séquence comportant $n$ unités $C V$ une séquence comportant $n+1$ unités $\mathrm{CV}$, que l'allongement soit vocalique (Figure 2) ou consonantique (Figure 3). L'évolution de la séquence MHA vater (> NHA V[a:]ter / fatter 'père') est représentée sous les Figures 2-3 ${ }^{14}$.

\footnotetext{
11. Excepté dans le cas du gouvernement infrasegmental, qui est une relation entre consonnes exclusivement.

12. Les positions vocaliques et consonantiques se trouvant sur des niveaux séparés, une position consonantique et une position vocalique ne pourront jamais être adjacentes à proprement parler.

13. i.e. les noyaux associés de manière permanente à une portion de mélodie, par opposition aux noyaux pleins non-stables, i.e. aux voyelles alternant avec $\emptyset$ (cf. Scheer, $2004: 661 s q q$.), dont la capacité à gouverner et/ou licencier varie d'une langue à l'autre.
}

14. Dans la Figure 2, l'unité CV additionnelle, absente en MHA mais présente en NHA, est encadrée. 


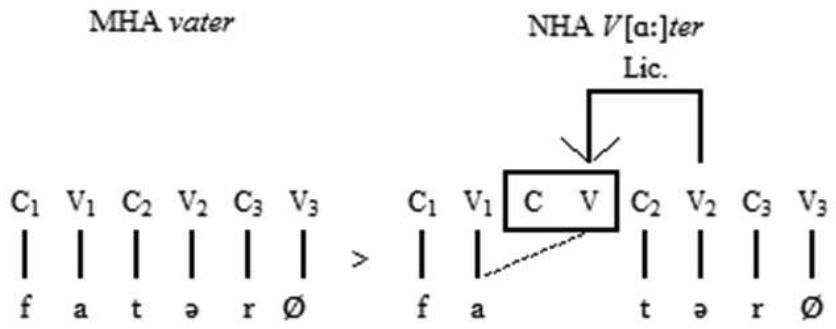

[a:]

Figure 2 : Allongement vocalique : MHA vater > NHA V[a:]ter 'père'

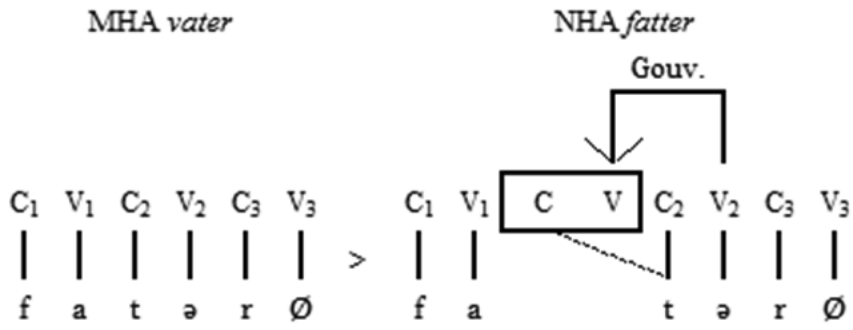

[t:]

Figure 3 : Allongement consonantique : MHA vater > NHA fatter 'père'

L'allongement requiert de l'espace syllabique inséré à droite de la voyelle tonique : celui-ci doit pouvoir être exploité par une voyelle (Figure 2) ou par une consonne (Figure 3), selon les cas. Pour recevoir une interprétation phonétique, la position vocalique supplémentaire $V_{+}$a besoin d'être licenciée (i.e. soutenue) par une position vocalique située à sa droite $\left(V_{2}\right.$ dans Figure 2$)$; pour que le $C V$ accentuel puisse être exploité par une position consonantique (Figure 3), $V_{+}$doit demeurer silencieuse et doit donc être gouvernée par un noyau situé à sa droite (ici $\mathrm{V}_{2}$ ).

Mais d'où vient l'espace syllabique additionnel nécessaire pour l'allongement constaté ? Cet espace syllabique n'étant disponible que sous l'accent - aucun allongement n'est en effet attesté dans les syllabes atones (cf. Tableau 2) -, il est légitime de postuler qu'il est la manifestation de l'accent tonique lui-même, i.e. que l'accent est interprété par la phonologie comme de l'espace syllabique. En d'autres termes, l'accent tonique correspond à une portion de la chaîne phonologique et est donc un objet connu de et totalement intégré à la composante phonologique: il ne s'agit pas d'un diacritique. Si l'on admet que l'accent 
se manifeste en phonologie comme de l'espace syllabique, le fait d'observer un allongement (vocalique ou consonantique) - plutôt qu'un abrégement - en syllabe tonique ${ }^{15} \mathrm{n}$ 'est plus le fait du hasard : l'espace syllabique additionnel en est la cause directe, une cause purement phonologique ${ }^{16}$.

Par ailleurs, l'espace syllabique additionnel ne peut être utilisé par une consonne ou par une voyelle qu'à condition que la séquence obtenue soit phonologiquement acceptable (cf. Larsen, 1998 : 90 ; Caratini, 2009 : 428 sqq.) : en allemand, l'allongement vocalique $n^{\prime}$ affecte en effet que les voyelles suivies maximalement d'une seule consonne (cf. Tableau 3) ${ }^{17}$; l'allongement consonantique ne concerne que les consonnes intervocaliques et finales immédiatement précédées de la voyelle tonique. Le CV accentuel ne peut être maintenu au sein de la séquence phonologique que s'il ne donne pas naissance à une chaîne agrammaticale sur le plan phonologique. En d'autres termes, la position vocalique additionnelle doit être gouvernée pour demeurer silencieuse (cf. Figure 3) ou licenciée pour pouvoir être associée à une portion de mélodie (cf. Figure 2).

15. Les grammaires diachroniques de l'allemand mentionnent également un processus d'abrégement vocalique lors de la transition entre le MHA et le NHA (cf. Paul et al., 2007 : $\$ 47$ ). Cependant, ce processus n'affecte pas uniquement les syllabes accentuées : l'abrégement a lieu, certes, sous l'accent dans certaines conditions de structure syllabique, mais également en position atone. L'abrégement vocalique n'est donc pas directement lié à l'accent.

16. L'idée de représenter l'accent tonique par de l'espace syllabique n'est cependant pas propre à la phonologie CVCV. D'autres auteurs, adeptes d'autres théories phonologiques (cf. Vogel 1982, qui évolue dans le cadre de la phonologie prosodique), proposent de représenter l'accent tonique par d'autres unités : un point squelettal (x, C ou V), une more, etc. La phonologie CVCV (cf. Larsen 1994, 1996, 1998 ; Scheer \& Szigetvári 2005) postule que l'accent se matérialise par une unité $\mathrm{CV}$, qui correspond à la plus petite unité syllabique pouvant être manipulée par la phonologie. La position du CV accentuel est spécifique à chaque langue : celui-ci peut être inséré à droite ou à gauche de la voyelle tonique (cf. Scheer, 2004 : § 224 ; Ségéral \& Scheer, 2008 : 504 sqq.) S'il est inséré à sa droite, comme dans le cas de la diachronie de l'allemand, il est attendu que sa présence sera perceptible sur la voyelle tonique ainsi que sur la consonne suivante ; s'il est inséré à sa gauche, sont attendus des effets sur l'initiale de la syllabe tonique (consonne) et sur la périphérie gauche de celle-ci.

17. La situation peut être différente dans d'autres langues : en coratin, dialecte italien parlé dans la région des Pouilles en Italie, l'unité CV additionnelle est maintenue dans la chaîne phonologique quel que soit le contexte syllabique dans lequel elle se trouve (cf. Bucci 2009). 
MHA

b. $\quad$ v $\circ 1 \quad \emptyset$

c. $\quad \mathrm{z}$ e $\partial \mathrm{n} \varnothing$

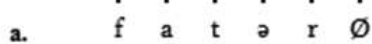

d.

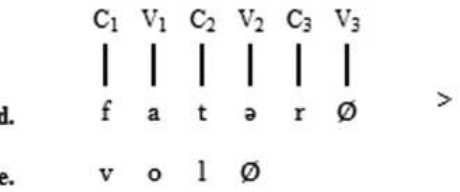

f.

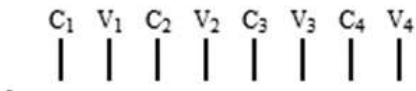

f. a $1 \varnothing$ t $\varnothing$

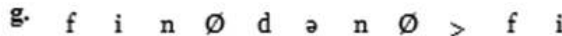
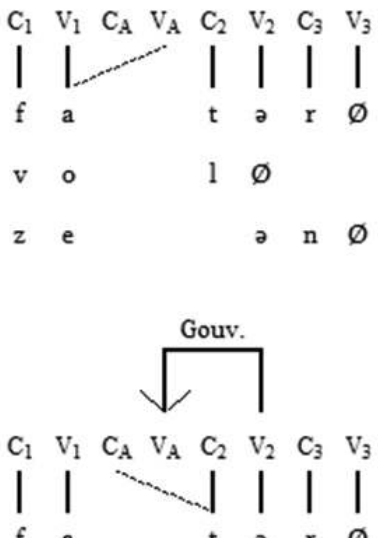

vo

$1 \varnothing$

Figure 4 : Allongement : contextes

Tableau 10 : Allongement vocalique vs Allongement consonantique

\begin{tabular}{|l|l|}
\hline Allongement vocalique & Allongement consonantique \\
\hline $\begin{array}{l}\text { a. MHA fater > NHA F[a:]ter 'père' } \\
\text { b. MHA wol > NHA } w[0:] h l \text { 'bien' }\end{array}$ & d. MHA fater > NHA fatter 'père' \\
c. MHA sehen > NHA s[e:]hen 'voir' & e. MHA wol > NHA woll 'bien' \\
\hline
\end{tabular}

Tableau 11 : Aucun allongement

Aucun allongement
f. MHA alt > NHA [a]/t 'vieux'
g. MHA finden > NHA $f[$ I]nden 'trouver'


Dans les exemples a. à c. supra, la position vocalique $\mathrm{V}_{\mathrm{A}}$ fournie par le $\mathrm{CV}$ accentuel est licenciée par le noyau suivant, ce qui lui permet d'être associée à une portion de mélodie : la voyelle tonique devient longue.

Dans les exemples d. et e., la position $\mathrm{V}_{2}$ gouverne $\mathrm{V}_{\mathrm{A}}$, ce qui permet à $\mathrm{V}_{\mathrm{A}}$ de rester non-exprimée : $c^{\prime}$ est donc la consonne suivante qui fait usage de l'espace syllabique fourni par l'accent : la consonne devient longue.

Dans les exemples f. et g., en revanche, la position vocalique fournie par l'accent ne peut être ni gouvernée ni licenciée car le gouverneur/licencieur potentiel est un noyau vide interne. Par conséquent, aucun allongement, ni consonantique ni vocalique, ne peut avoir lieu et l'unité CV accentuelle ne peut être maintenue dans la chaîne phonologique.

Si l'on considère la diachronie récente de l'allemand, il est donc possible de représenter l'accent comme de l'espace syllabique inséré à droite de la voyelle tonique.

\subsection{Synchronie de l'allemand moderne}

En allemand moderne, l'accent affecte principalement l'initiale des syllabes toniques : un coup de glotte est inséré à l'initiale des syllabes accentuées commençant par une voyelle, et les occlusives non-voisées sont aspirées lorsqu'elles se trouvent à l'initiale d'une syllabe accentuée (§ 3.2). Cela signifie qu'en allemand moderne, contrairement à ce qui a été observé pour la diachronie de la langue, le CV accentuel est inséré à la gauche de la voyelle accentuée. Il y a donc rupture entre la situation diachronique (insertion du $\mathrm{CV}$ accentuel à droite de la voyelle tonique) et la situation synchronique (insertion du CV accentuel à gauche de la voyelle tonique).

Nous verrons comment concilier cela avec le fait que l'accent a également un effet sur les voyelles toniques (§ 3.2.3), i.e. sur la partie droite des syllabes toniques.

\subsubsection{Attaques}

Il a été démontré supra $(\S 3.2 .1)$ qu'un coup de glotte peut être inséré en position initiale de syllabes commençant par une voyelle, à condition que cellesci soient toniques (ex. The[?]ater 'théâtre') et/ou se trouvent à l'initiale absolue de mot (ex. [?]enttäuschen 'décevoir'). La phonologie CVCV propose une analyse unifiée du phénomène basée sur la présence d'une unité CV marquant l'initiale absolue de mot (cf. Lowenstamm 1999). Selon T. Scheer (2000 : 153), un coup de glotte remplit toute attaque vide non-gouvernée (cf. Figure 5). La position $C_{2}$ n'étant pas gouvernée par le noyau suivant $\left(V_{2}\right.$ doit en effet gouverner $\left.V_{A}\right)$, un coup de glotte est inséré sous $C_{2}$ dans l'exemple a. Dans l'exemple b., $V_{2}$ n'est pas contraint de gouverner un noyau précédent : le gouvernement qu'il exerce se porte donc sur $C_{2}$ : un coup de glotte ne peut donc faire surface à cet endroit. 
a.

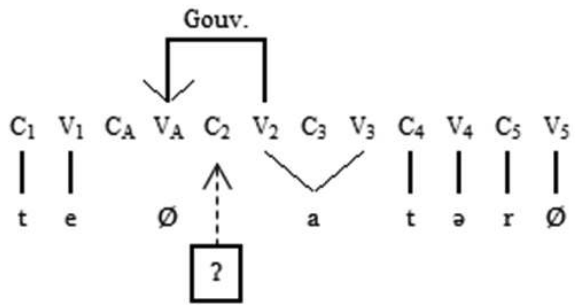

b.

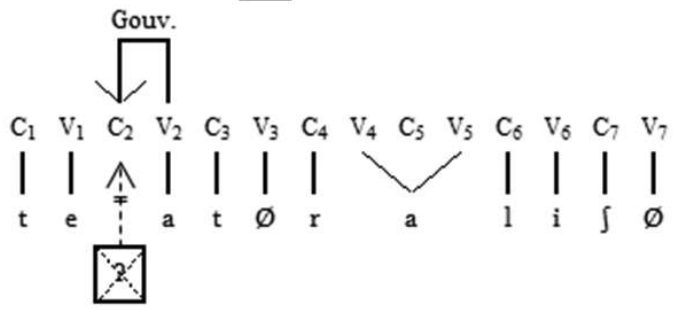

Figure 5 : Insertion du coup de glotte sous l'accent

La même analyse peut être étendue au contexte du début de mot (cf. Figure 6) :

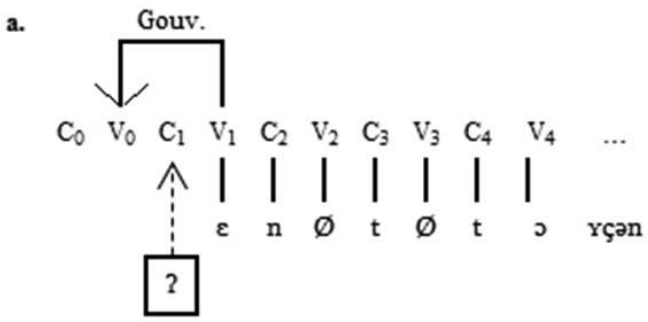

[?]ent+täuschen « décevoir»

b.

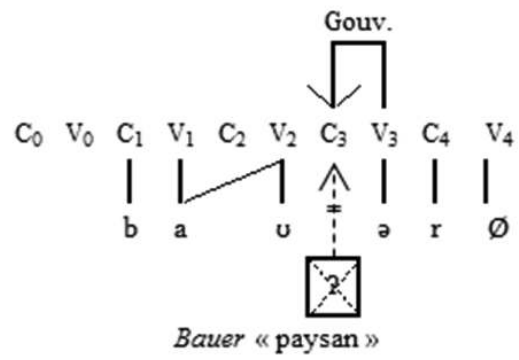

Figure 6 : Insertion du coup de glotte en début de mot $^{\text {a }}$

a. $\mathrm{C}_{0} \mathrm{~V}_{0}$ correspond au $\mathrm{CV}$-initial. 
Dans l'exemple a. (Figure 6), $\mathrm{V}_{1}$ doit licencier la position vocalique du CV initial et ne peut donc gouverner $C_{1}$, qui reste donc vide et non-gouvernée : un coup de glotte est inséré. Dans l'exemple b., $V_{3}$ n'a nul besoin de gouverner un noyau précédent ; il gouverne donc $C_{3}$ qui ne peut donc accueillir un coup de glotte.

Une analyse similaire de l'aspiration des occlusives non-voisées en début de mot et à l'initiale de syllabes accentuées est envisagée par T. Scheer (2000: 143). Sa proposition, qui rend la présence d'une unité $\mathrm{CV}$ vide à la gauche des consonnes concernées responsable de l'aspiration, concerne en réalité l'aspiration des occlusives non-voisées en anglais ; mais l'aspiration ayant lieu dans des contextes identiques en anglais et en allemand, elle peut être adaptée aux données de l'allemand (cf. Figure 7).

a.

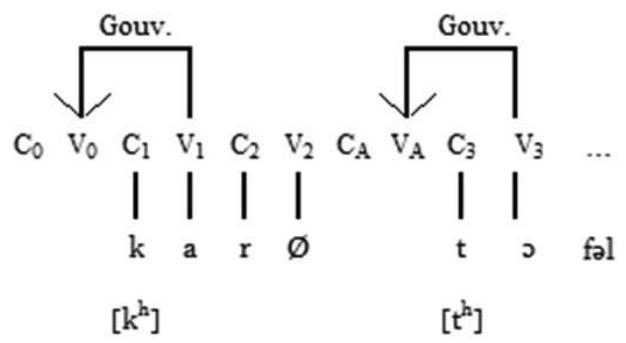

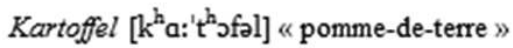

b.

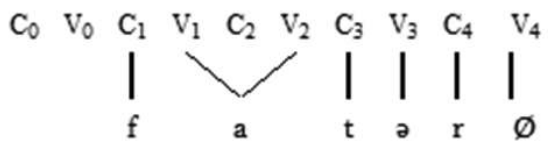

$[\mathrm{t}]$

Vater ['fa:tə] « père »

Figure 7 : Aspiration des occlusives non-voisées

Dans l'exemple a., la consonne initiale, précédée immédiatement d'une unité CV vide (début de mot), est aspirée, tout comme la consonne suivant immédiatement le CV accentuel. Dans l'exemple b., en revanche, l'occlusive non-voisée n'étant pas précédée d'une unité $C V$ vide, elle ne peut être aspirée. 
Il n'est possible de rendre compte de l'occurrence du coup de glotte ainsi que de l'aspiration des occlusives non-voisées en allemand en début de mot et à l'initiale de syllabes accentuées que si l'on postule que le CV accentuel est inséré à gauche de la voyelle accentuée ${ }^{18}$. Sans ce postulat, $l^{\prime}$ aspiration $/ l^{\prime}$ occurrence du coup de glotte en début de mot et à l'initiale de syllabes accentuées ne pourrait être unifiée.

\subsubsection{Expression phonétique de la longueur (phonologique) vocalique}

Le CV accentuel étant inséré à gauche de l'unité CV comprenant la voyelle tonique, il ne peut avoir d'influence sur cette dernière. Les données présentées (§ 3.2.3) posent donc problème : en effet, parmi les voyelles phonologiquement longues, seules celles qui sont accentuées sont phonétiquement longues ; les autres, atones, sont phonétiquement brèves. Les données présentées supra (§ 3.2.3) sont rappelées dans le Tableau 12 :

\section{Tableau 12 : Réalisation phonétique des voyelles phonologiquement longues}

\begin{tabular}{|l|l|}
\hline Voyelle sous l'accent & Voyelle hors accent \\
\hline Möbel ['mø:bəl] 'meuble' & möbl+ieren [mø'bli::əən] 'meubler' \\
\hline Theater [the'Ta:te] 'theâtre' & theatr+alisch [thea't $\chi a: I I]$ 'théâtral' \\
\hline Zug ['țu:k] 'train' & An+zug ['antsuk] 'costume' \\
\hline Motor ['mo:toø] 'moteur' & Motor+en [mo'tho::əən] 'moteurs' \\
\hline
\end{tabular}

Une analyse basée sur l'idée que l'accent se manifesterait simultanément par deux unités CV - une étant responsable de l'occurrence du coup de glotte et de l'aspiration des occlusives non-voisées et l'autre de l'occurrence des voyelles phonétiquement longues - n'est pas défendable pour deux raisons. D'une part, la phonologie ne peut manipuler, maximalement, qu'une seule unité CV ; cela est en effet un des postulats de départ de la phonologie CVCV. D'autre part, un second $\mathrm{CV}$ accentuel inséré à droite des voyelles toniques poserait problème. La longueur vocalique, en allemand moderne, est lexicalisée : 217 paires minimales sont recensées dans E. Caratini (2009), et nombreuses sont les formes dans

18. Il est un fait que les occlusives non-voisées de l'allemand, ancien comme moderne, ne sont pas de même nature que celles du français ou de l'italien : une différence est traditionnellement faite entre les lenis et les fortis (cf. Paul et la., 2007 : § 83-124). Mais la situation attestée en VHA et en MHA semble différente de celle attestée en NHA. En effet, si les occlusives du VHA et du MHA peuvent être réparties en deux groupes (fortis vs. lenis) comme c'est le cas en NHA, les consonnes du type fortis du VHA et du MHA semblent ne pas avoir hérité leurs caractéristiques articulatoires du contexte dans lequel elles se trouvaient. En NHA, en revanche, l'aspiration des occlusives non-voisées n'a lieu que dans deux contextes : en début de mot et à l'initiale de syllabe accentuée. Il s'agit donc en NHA d'une aspiration liée à la position des consonnes concernées dans la chaîne phonique, et non d'une propriété stable des occlusives non-voisées comme c'était sans doute le cas en VHA et en MHA. Par ailleurs, le VHA, le MHA et le NHA ayant des systèmes phonologiques distincts, il n'y a aucune raison de postuler que l'aspiration dans ces trois langues soit due au(x) même(s) mécanisme(s) : la force relative (aspiration ?) des occlusives non-voisées était vraisemblablement lexicale (i.e. non dérivée) et ne nécessitait donc pas la présence d'un $\mathrm{CV}$ accentuel positionné à gauche de la voyelle tonique et d'un $\mathrm{CV}$ marquant le début de mot en VHA et en MHA ; elle semble, en revanche, être dérivée (i.e. due à des facteurs positionnels et accentuels qui se traduisent, sur le plan phonologique, par l'insertion d'unités CV) en NHA. 
lesquelles une voyelle longue précède un groupe consonantique (ex. fahnden ['fa:ndən] 'rechercher', Obst ['Po:pst] 'fruits', etc.). Cela signifie que la longueur vocalique n'est plus contrainte en allemand moderne comme elle le fut dans la transition entre le MHA et le NHA. Si un second CV accentuel était inséré à la droite de chaque voyelle tonique, on s'attendrait à ce que chaque voyelle brève puisse être longue sous l'accent, ce qui n'est pas le cas : la voyelle de Mitte 'milieu' est brève qu'elle reçoive l'accent primaire ou non, tout comme celle de Amt 'service'.

Si un second CV accentuel ne peut être la cause de la variation mentionnée supra, c'est que l'accent, en allemand moderne, ne se manifeste pas seulement sous la forme d'espace syllabique. Dans le cas qui nous intéresse ici, il ne fait en réalité que supporter l'expression phonétique de la longueur phonologique : une voyelle phonologiquement longue n'est réalisée comme telle sur le plan phonétique que si elle y est autorisée par la présence de l'accent tonique (ex. Möbel ['mø:bəl] 'meuble' vs. möblieren [mø'bli::ən] 'meubler') : l'accent tonique promeut l'expression, au niveau phonétique, de la longueur phonologique. Cette manifestation-là de l'accent ne peut correspondre à de l'espace syllabique et est donc à considérer comme diacritique $\left(/^{\prime} /\right)$ : elle se limite à promouvoir la réalisation phonétique de la longueur phonologique.

\section{CONCLUSION}

En prenant appui sur l'étude de cinq phénomènes phonologiques dans l'accomplissement desquels l'AT jour un rôle essentiel, nous avons proposé deux représentations de l'accent - une représentation syllabique (une unité CV insérée à droite ou à gauche de la voyelle tonique, cf. Scheer 2000) et une représentation diacritique rendant licite l'expression phonétique $d^{\prime}$ 'un caractère phonologique pouvant cohabiter au sein d'une langue donnée. Si tout cela est exact, nous nous attendons à ce que deux types de processus différents puissent être associés à ces deux formes distinctes d'accent tonique.

Par ailleurs, si la substance de l'AT est claire et tangible dans sa manifestation syllabique (une unité $\mathrm{CV}$ est un objet phonologique bien défini qui existe indépendamment de la manifestation syllabique de l'AT), celle de l'AT diacritique reste plus subtile car difficile à cerner. L'étude d'autres phénomènes phonologiques liés à l'AT en général et à l'AT diacritique en particulier reste donc nécessaire dans l'optique de mieux comprendre sa substance.

\section{Références}

ALBER B. (2001), "Regional variation and edges: glottal stop epenthesis and dissimilation in Standard and Southern varieties of German", Zeitschrift für Sprachwissenschaft 20 (1), 3-41.

Bluhme H. (1970), " Das phonologische System des Deutschen ", Lingua 25, 358-80. 
Bourciez E. (1910), Précis de phonétique historique du français, Paris : Klincksieck.

Bourciez E. \& Bourciez J. (1967), Phonétique française. Étude historique, Paris : Klincksieck. Braune W. \& ReIfFEnSteIn I. (2004), Althochdeutsche Grammatik, Tübingen : Niemeyer.

Buccı J. (2009), Réduction vocalique et partage mélodique en coratin, Mémoire de Master, Université Nice Sophia Antipolis.

CARATINI E. (2009), Vocalic and consonantal quantity in German: synchronic and diachronic perspectives, Thèse de l'Université de Nice et de I'Universität Leipzig.

Carvalho J. Brandão de, Nguyen N. \& Wauquier S. (2010), Comprendre la phonologie, Paris : Presses Universitaires de France.

Chomsky N. \& Halle M. (1968), The Sound Pattern of English, New York: Harper \& Row.

Coluinge N. E. ([1985] 1996), The laws of Indo-European, Amsterdam: John Benjamins.

CRYSTAL D. ([1987] 1994]), The Cambridge Encyclopedia of Language, Cambridge: Cambridge University Press.

D'InTRONo F. \& WeSton R. (1997), " Preservazione, Cambio e Riduzione Vocalica in Coratino: Effetti e Strategia dell'OCP ", in L. Agostini (ed.), Atti de terzo convegno della Società internazionale di linguistica e filologia italiana, Perugia : Edizioni scientifiche italiane, 287302.

FónAGY I. (1979), "Gamme sémantique des coups de glotte dans le français moderne ", Le français dans le monde 143, 43-60.

GIEGERICH H. J. (1985), Metrical phonology and phonological structure - German and English, Cambridge: Cambridge University Press.

Goldsmith J. A., Riggle J. \& Yu A. C. L. ([2011] 2014), The Handbook of Phonological Theory, Malden (MA)/Oxford: Wiley Blackwell.

GUSSENHOVEN C. (1986), “English plosive allophones and ambisyllabicity”, Gramma - Nijmeegs tijdschrift voor taalkunde 10, 119-141.

HALL T. A. (1992), Syllable structure and syllable-related processes in German, Tübingen: Niemeyer.

Hall T. A. (2000), Phonologie : eine Einführung, Leipzig : W. de Gruyter.

HARRIS J. (1994), English sound structure, Oxford: Blackwell.

HAYES B. P. (1990), "Precompiled phrasal phonology", in S. Inkelas \& D. Zec (eds), The Phonology-syntax Connection, Chicago: University of Chicago Press, 85-108.

IVERSON G. K. \& SALMONS J. C. (1995), "Aspiration and laryngeal representation in Germanic", Phonology 12 (3), 369-396.

JESSEN M. (1998), Phonetics and Phonology of the tense and lax obstruents in German, Amsterdam: Benjamins.

KAHN D. (1976), Syllable-based Generalizations in English Phonology, Cambridge (MA): The MIT Press.

KAYE J. D. (1990), “Coda licensing”, Phonology 7, 301-330.

Kaye J. D., Lowenstamm J. \& Vergnaud J.-R. (1990), “Constituent structure and government in phonology", Phonology 7, 193-231.

KENSTOWICZ M. \& KISSEBERTH C. (1979), Generative phonology: description and theory, San Diego: Academic Press.

LADD D. R. (ed.) (1996), Intonational phonology, Cambridge: Cambridge University Press.

LADEFOGED P. (2001), Vowels and consonants: an introduction to the sounds of languages, Oxford: Blackwell. 
LARSEN B. U. (1994), Some aspects of vowel length and stød in Danish, Thèse de I'Université Paris 7.

LARSEN B. U. (1996), Longueur vocalique, raddoppiamento sintattico et l'article défini, Ms.

LARSEN B. U. (1998), "Vowel Length, Raddoppiamento Sintattico and the selection of the definite article in Italian", in P. Sauzet (éd.), Langues et Grammaires, II-III, Phonologie, Paris : Université Paris 8, 87-102.

LIBERMAN M. \& PRINCE A. (1977), “On stress and linguistic rhythm”, Linguistic Inquiry 8, 249-336.

LoWEnStAMm J. (1996), “CV as the only syllable type”, in J. Durand \& B. Laks (eds), Current Trends in Phonology - Models and Methods, Salford (UK): European Studies Research Institute, 419-441.

LoWENSTAMm J. (1999), “The beginning of the word”, in J. Rennison \& K. Kühnhammer (eds), Syllables!? - Phonologica 1996, The Hague: Holland Academic Graphics, 153-166.

MeRTENS P. (2009), "Prosodie, syntaxe et discours : autour d'une approche prédictive ", in H.-Y. Yoo \& E. Delais-Roussarie (éds), Interface Discours \& Prosodie - IDP 2009, 19-32. [http://makino.linguist.jussieu.fr/idp09/actes_fr.html]

Minkova D. (1982), “The environment for Open Syllable Lengthening in Middle English”, Folia Linguistica Historica 3 (2), 29-58.

Moulton W. G. (1947), “Juncture in Modern Standard German”, Language 23, 212-226.

MÜLLER W. \& ZARNCKE F. ([1871] 2007), Mittelhochdeutsches Wörterbuch - mit Benutzung des Nachlasses von G. F. Benecke ausgearbeitet, Stuttgart : Hirzel.

Nespor M. \& Vogel I. B. ([1986] 2007), Prosodic phonology, Berlin/New York: Mouton de Gruyter.

PAUL H. O. T. ([1916] 1968), Deutsche Grammatik, Tübingen : Niemeyer.

PAUL H. O. T. (1884), "Beiträge zur Geschichte der Lautentwicklung und Formenassoziation 11. Vokaldehnung und Vokalverkürzung im Neuhochdeutschen ", Beiträge zur Geschichte der Deutschen Sprache und Literatur 9, 101-34.

PAUL H. O. T. et al. ([1881] 2007), Mittelhochdeutsche Grammatik, Tübingen : Niemeyer.

Reetz H. \& Jongman A. (2009), Phonetics - Transcription, production, acoustics and perception, Oxford: Wiley Blackwell.

RITZERT A. (1898), "Die Dehnung der mhd. kurzen Stammsilbenvokale in den Volksmundarten des hochdeutschen Sprachgebiets auf Grund der vorhandenen Dialektliteratur ", Beiträge zur Geschichte der Deutschen Sprache und Literatur 23, 131-222.

ROACH P. (2001), Phonetics, Oxford: Oxford University Press.

Russ C. V. J. (1969), "Die Ausnahmen zur Dehnung der mhd. Kurzvokale in offener Silbe ", Zeitschrift für Dialektologie und Linguistik 36, 82-88.

SCHEER T. (2000), De la localité, de la morphologie et de la phonologie en phonologie, Habilitation à Diriger des Recherches de l'Université de Nice.

SCHEER T. (2004), A Lateral Theory of Phonology, Vol. 1: What is CVCV and why should it be?, Berlin: Mouton de Gruyter.

SCHEER T. (2005), "Postcard theory: how higher level modules communicate with phonology through a translator's office", Paper presented at the 36th Poznań Linguistic Meeting - PLM 2005, Poznań.

SCHEER T. (2011), A Guide to Morphosyntax-Phonology Interface Theories. How Extra-Phonological Information is Treated in Phonology since Trubetzkoy's Grenzsignale, Berlin: Mouton de Gruyter. 
SCHEeR T. \& SzIgetVÁRI P. (2005), “Unified representations for stress and the syllable", Phonology 22, 37-75.

SCHIRMUNSKI V. M. ([1956] 1962), Deutsche Mundartkunde: vergleichende Laut- und Formenlehre der deutschen Mundarten, Berlin: Akademie-Verlag.

Ségéral P. \& Scheer T. (2001), "La coda-miroir ", Bulletin de la Société Linguistique de Paris XCVI (1), 107-152.

Ségéral P. \& Scheer T. (2008), "The coda mirror, stress and positional parameters", in P. Ségéral, J. Brandão de Carvalho \& T. Scheer (eds), Lenition and fortition, Berlin: Mouton de Gruyter, 483-518.

SEILER G. (2004), "How contrastive vowel quantity can become non contrastive", in N. Adams et al. (eds), Proceedings from the Annual Meeting of the Chicago Linguistic Society 40 (1), Chicago: Chicago Linguistic Society, 103-129.

SEILER G. (2009), "Sound change or analogy? Monosyllabic lengthening in German and some of its consequences", Journal of Comparative Germanic Linguistics 12, 229-272.

Thomas J. M.-C., BouquiAux L. \& CLoAREC-HeISS F. (1976), Initiation à la phonétique : phonétique articulatoire et phonétique distinctive, Paris : Presses Universitaires de France.

VERNER K. (1877), "Eine Ausnahme der ersten Lautverschiebung ", Zeitschrift für vergleichende Sprachwissenschaft 23 (2), 97-130.

VoGEL I. B. (1982), La Sillaba come unità fonologica, Bologna : Zanichelli.

WIESE R. (1996), The Phonology of German, New York: Oxford University Press. 